\title{
The Average Sensitivity of Bounded-Depth Formulas
}

\author{
Benjamin Rossman*
}

June 12, 2018

\begin{abstract}
We show that unbounded fan-in boolean formulas of depth $d+1$ and size $s$ have average sensitivity $O\left(\frac{1}{d} \log s\right)^{d}$. In particular, this gives a tight $2^{\Omega\left(d\left(n^{1 / d}-1\right)\right)}$ lower bound on the size of depth $d+1$ formulas computing the PARITY function. These results strengthen the corresponding $2^{\Omega\left(n^{1 / d}\right)}$ and $O(\log s)^{d}$ bounds for circuits due to Håstad (1986) and Boppana (1997). Our proof technique studies a random process where the Switching Lemma is applied to formulas in an efficient manner.
\end{abstract}

\section{Introduction}

We consider boolean circuits with unbounded fan-in AND and OR gates and negations on inputs. Formulas are the class of tree-like circuits in which all gates have fan-out 1. Size of circuits (including formulas) is measured by the total number of gates. Depth is the maximum number of gates on an input-to-output path.

Lower bounds against bounded-depth circuits were first proved in the 1980s [1, 3, 8, 4, culminating in a tight size-depth tradeoff for circuits computing the PARITY function. The technique, based on random restrictions, applies more generally to boolean functions with high average sensitivity.

Theorem 1 (Håstad [4]). Depth $d+1$ circuits computing PARITY have size $2^{\Omega\left(n^{1 / d}\right)}$.

Theorem 2 (Boppana [2]). Depth $d+1$ circuits of size $s$ have average sensitivity $O(\log s)^{d}$.

In this paper, we prove stronger versions of these results for bounded-depth formulas:

Theorem 3. Depth $d+1$ formulas computing PARITY have size $2^{\Omega\left(d\left(n^{1 / d}-1\right)\right)}$.

Theorem 4. Depth $d+1$ formulas of size s have average sensitivity $O\left(\frac{1}{d} \log s\right)^{d}$.

Theorems 3 and 4 directly strengthen Theorems 1 and 2 in light of the following

Fact 5. Every depth $d+1$ circuit of size $s$ is equivalent to a depth $d+1$ formula of size at most $s^{d}$.

Theorems 1, 2, 3, 4) are asymptotically tight, since PARITY is computable by depth $d+1$ circuits (resp. formulas) of size $n 2^{O\left(n^{1 / d}\right)}\left(\right.$ resp. $\left.2^{O\left(d\left(n^{1 / d}-1\right)\right)}\right)$.

${ }^{*}$ National Institute of Informatics (Tokyo, Japan) and Simons Institute (Berkeley, CA). rossman@nii.ac.jp 
The main tool in the proof of Theorems1] and[2] is Håstad's Switching Lemma [4]. The Switching Lemma states that every small-width CNF or DNF simplifies, with high probability under a random restriction, to a small-depth decision tree. This yields lower bounds against bounded-depth circuits via a straightforward depth-reduction argument. In this paper we show how the Switching Lemma can be applied more efficiently to bounded-depth formulas, though in a less straightforward manner.

In more detail: for independent uniformly distributed random $\sigma \in\{0,1\}^{n}$ ("assignment") and $\tau \in[0,1]^{n}$ ("timestamp"), we consider the family of restrictions $\left\{R_{p}^{\sigma, \tau}\right\}_{0 \leq p \leq 1}$ (i.e. functions $[n] \rightarrow\{0,1, *\}$ representing partial assignments to input variables $\left.x_{1}, \ldots, x_{n}\right)$ where $R_{p}^{\sigma, \tau}$ sets the variable $x_{i}$ to $\sigma_{i}$ if $\tau_{i}<p$ and leaves $x_{i}$ unset if $\tau_{i} \geq p$. In the usual application of the Switching Lemma to circuits of depth $d+1$, all subcircuits of depth $k+1$ are hit with the restriction $R_{p_{k}}^{\sigma, \tau}$ for a fixed sequence $p_{1}>\cdots>p_{d}$ (typically $\left.p_{k}=n^{-k /(d+1)}\right)$. In this paper we achieve sharper bounds against formulas by hitting each subformula $\Phi$ with the restriction $R_{\mathbf{q}(\Phi)}^{\sigma, \tau}$ where the parameter $\mathbf{q}(\Phi)$ $\left(=\mathbf{q}^{\sigma, \tau}(\Phi)\right)$ is defined inductively, according to a random process indexed by subformulas of $\Phi$. Our technical main theorem is a tail bound on $\mathbf{q}(\Phi)$, viewed as a random variable determined by $\sigma$ and $\tau$.

After preliminary definitions in $₫ 2$, we state and prove our technical main theorem in $₫ 3$ and $₫ 4$, As a corollaries, we derive Theorem 3 in $\$ 5$ and Theorem 4 in $\$ 6$, In $\$ 7$ we state a further corollary of our results on the relative power of formulas vs. circuits.

\section{Preliminaries}

$\mathbb{N}=\{0,1,2, \ldots\} \cdot[n]=\{1, \ldots, n\} \cdot \exp (\lambda)=\mathrm{e}^{\lambda}$.

\section{$2.1 \quad$ Formulas}

A formula is a finite rooted tree whose leafs ("inputs") are labeled by literals (i.e. variables $x_{i}$ or negated variables $\neg x_{i}$ ) and whose non-leafs ("gates") are labeled by AND or OR. (Gates have unbounded fan-in.) Every formula $\Phi$ computes a boolean function on the same set of variables.

The size of a formula $\Phi$, denoted by $|\Phi|$, is the number of gates in $\Phi$. (Note that every lower bound on size is also a lower bound on leafsize, i.e., the number of leaves in a formula.) The depth of $\Phi$ is the maximum number of gates on an input-to-output path. Formulas of depth 0 are literals; formulas of depth 1 are clauses (i.e. an AND or OR of literals). We are often interested in formulas of depth $\geq 2$ and speak of "depth $d+1$ " where $d$ is an arbitrary positive integer.

\subsection{Boolean functions and restrictions}

A restriction is a function $\varrho:[n] \rightarrow\{0,1, *\}$, viewed as a partial assignment of boolean input variables $x_{1}, \ldots, x_{n}$ to 0,1 or $*$ (meaning "unset"). For a boolean function $f:\{0,1\}^{n} \rightarrow\{0,1\}$, the restricted function $f\left\lceil\varrho:\{0,1\}^{\varrho^{-1}(*)} \rightarrow\{0,1\}\right.$ is defined in the usual way. For $p \in[0,1]$, we write $\mathcal{R}_{p}$ for the distribution on restrictions $\varrho$ where $\mathbb{P}[\varrho(i)=*]=p$ and $\mathbb{P}[\varrho(i)=0]=\mathbb{P}[\varrho(i)=1]=$ $(1-p) / 2$ independently for all $i \in[n]$.

\subsection{Average sensitivity and decision-tree depth}

The average sensitivity as $(f)$ of a boolean function $f$ is the expected number of input bits that, when flipped, change the output of $f$, starting with a random input assignment. 
The decision-tree depth $\mathrm{D}(f)$ of $f$ is the minimum depth of a decision tree which computes $f$; in particular, $\mathrm{D}(f)=0$ iff $f$ is constant. Two elementary facts which we will use later (see [2]): for every boolean function $f$,

$$
\begin{aligned}
\operatorname{as}(f) & \leq \mathrm{D}(f) & & \text { (i.e. average sensitivity is at most decision-tree depth), } \\
\underset{\varrho \sim \mathcal{R}_{p}}{\mathbb{E}}[\operatorname{as}(f\lceil\varrho)] & =p \cdot \operatorname{as}(f) & & \text { for all } 0 \leq p \leq 1 .
\end{aligned}
$$

Håstad's Switching Lemma relates random restrictions and decision-tree depth. We give a somewhat nonstandard statement (the usual statement is in terms of width- $k$ CNFs and width- $\ell$ DNFs).

Lemma 6 (Switching Lemma [4]). Let $k, \ell \in \mathbb{N}$. Suppose $f$ is the AND or OR of an arbitrary family $\left\{f_{i}\right\}$ of boolean functions with $\mathrm{D}\left(f_{i}\right) \leq k$ for all $i$. Then for all $0 \leq p \leq \frac{1}{2}$,

$$
\underset{\varrho \sim \mathcal{R}_{p}}{\mathbb{P}}\left[\mathrm{D}(f\lceil\varrho) \geq \ell] \leq(5 p k)^{\ell} .\right.
$$

\section{A random process associated with formulas}

Definition 7. Let $\sigma \in\{0,1\}^{n}$ ("assignment") and $\tau \in[0,1]^{n}$ ("timestamp") be independent uniformly distributed random variables. For $0 \leq p \leq 1$, let $R_{p}^{\sigma, \tau}:[n] \rightarrow\{0,1, *\}$ be the restriction

$$
R_{p}^{\sigma, \tau}(i):= \begin{cases}\sigma_{i} & \text { if } \tau_{i}>p \\ * & \text { if } \tau_{i} \leq p\end{cases}
$$

We regard the family of restrictions $\left\{R_{p}^{\sigma, \tau}\right\}_{0 \leq p \leq 1}$ as a stochastic process where the parameter $p$ represents a "time" which starts at 1 and decreases to 0 . At the initial time $p=1$, the assignment $\sigma$ is fully masked (i.e. $R_{1}^{\sigma, \tau}$ is all $*$ 's). As $p$ decreases, the values of $\sigma$ are gradually unmasked, until the final time $p=0$ when $\sigma$ is fully revealed (i.e. $R_{0}^{\sigma, \tau}=\sigma$ ). Of course, for any fixed $p, R_{p}^{\sigma, \tau}$ is simply a random restriction with distribution $\mathcal{R}_{p}$.

Definition 8 (Main Definition). For all formulas $\Phi$, we define the "stopping time" $\mathbf{q}^{\sigma, \tau}(\Phi) \in[0,1]$ by the following induction:

- If $\Phi$ has depth 0 (i.e. $\Phi$ is a variable or negated variable), then $\mathbf{q}^{\sigma, \tau}(\Phi):=1$.

- If $\Phi$ is $\operatorname{AND}\left(\Psi_{1}, \ldots, \Psi_{m}\right)$ or $\operatorname{OR}\left(\Psi_{1}, \ldots, \Psi_{m}\right)$, then

$$
\mathbf{q}^{\sigma, \tau}(\Phi):=\frac{\mathbf{p}^{\sigma, \tau}(\Phi)}{14 \cdot \mathbf{k}^{\sigma, \tau}(\Phi)}
$$

where $\quad \mathbf{p}^{\sigma, \tau}(\Phi):=\min _{i} \mathbf{q}^{\sigma, \tau}\left(\Psi_{i}\right), \quad \mathbf{k}^{\sigma, \tau}(\Phi):=\max \left\{1, \max _{i} \mathrm{D}\left(\Psi_{i}\left\lceil R_{\mathbf{p}^{\sigma, \tau}(\Phi)}^{\sigma, \tau}\right)\right\}\right.$.

For the sake of readability, we will suppress $\sigma$ and $\tau$ whenever possible and simply write $\mathbf{q}(\Phi)$, $\mathbf{p}(\Phi), \mathbf{k}(\Phi)$. However, the reader should keep in mind that these random variables are determined, for all formulas $\Phi$, by a single pair of $\sigma$ of $\tau$. (We will continue to write $\sigma$ and $\tau$ when referring to restrictions $R_{p}^{\sigma, \tau}$.)

We view $\mathbf{q}(\Phi)$ as the stopping time for a stochastic process indexed by formulas $\Phi$. For $\Phi$ of depth $0, \mathbf{q}(\Phi)$ is the initial time 1 (when all variables are masked). For $\Phi$ of depth $\geq 1, \mathbf{q}(\Phi)$ is defined in terms of two auxiliary parameters: 
- $\mathbf{p}(\Phi)$ is the most advanced (i.e. minimum) stopping time $\mathbf{q}(\Psi)$ among children $\Psi$ of $\Phi$.

- $\mathbf{k}(\Phi)$ is the maximum decision-tree depth among children $\Psi$ of $\Phi$ upon being hit with the restriction $R_{\mathbf{p}(\Phi)}^{\sigma, \tau}$. (For technical reasons, we set $\mathbf{k}(\Phi)=1$ in the event that $D\left(\Psi\left\lceil R_{\mathbf{p}(\Phi)}^{\sigma, \tau}\right)=0\right.$ for all $\Psi$.)

If $\Phi$ is an AND (resp. OR), then $\Phi\left\lceil R_{\mathbf{p}(\Phi)}^{\sigma, \tau}\right.$ is a $\mathbf{k}(\Phi)$-CNF (resp. DNF). The choice of definition $\mathbf{q}(\Phi)=\mathbf{p}(\Phi) / 14 \cdot \mathbf{k}(\Phi)$ allows us to apply the Switching Lemma to $\Phi\left\lceil R_{\mathbf{p}(\Phi)}^{\sigma, \tau}\right.$. This is made precise by the following lemma. (Since the dependence on $\sigma$ and $\tau$ is crucial here, we use explicit notation: $\mathbf{q}^{\sigma, \tau}(\Phi)$, etc.)

Lemma 9. Let $\Phi$ be a formula of depth $\geq 1$ and let $q \in \operatorname{Supp}\left(\mathbf{q}^{\sigma, \tau}(\Phi)\right.$ ) (i.e. $q=\mathbf{q}^{\sigma, \tau}(\Phi)$ for some $\sigma \in\{0,1\}^{n}$ and $\left.\tau \in[0,1]^{n}\right)$. Then for all $0 \leq \alpha \leq 1$ and $\ell \in \mathbb{N}$,

$$
\underset{\sigma, \tau}{\mathbb{P}}\left[\mathrm{D}\left(\Phi\left\lceil R_{\alpha q}^{\sigma, \tau}\right) \geq \ell \mid \mathbf{q}^{\sigma, \tau}(\Phi)=q\right] \leq\left(\frac{\alpha}{\mathrm{e}}\right)^{\ell}\right.
$$

Proof. Fix $\Phi$ and $q$ as in the hypothesis of the lemma. Since $\Phi$ has depth $\geq 1$, it is the AND or OR of formulas $\Psi_{i}$. Let

$$
I:=\left\{(p, \varrho, k): \begin{array}{l}
q=p / 14 k \text { and there exist } \sigma \in\{0,1\}^{n} \text { and } \tau \in[0,1]^{n} \\
\text { such that } \mathbf{p}^{\sigma, \tau}(\Phi)=p, R_{p}^{\sigma, \tau}=\varrho \text { and } \mathbf{k}^{\sigma, \tau}(\Phi)=k
\end{array}\right\} .
$$

Note that $I$ is nonempty and indexes a partition of the event $\left\{\mathbf{q}^{\sigma, \tau}(\Phi)=q\right\}$ into subevents $\left\{\mathbf{p}^{\sigma, \tau}(\Phi)=p, R_{p}^{\sigma, \tau}=\varrho\right.$ and $\left.\mathbf{k}^{\sigma, \tau}(\Phi)=k\right\}$.

To prove the lemma, consider any $(p, \varrho, k) \in I$. Conditioning on this subevent, we can view $R_{\alpha q}^{\sigma, \tau}$ as the composition of $\varrho$ and an independent random restriction $\theta \sim \mathcal{R}_{\alpha / 14 k}$. Since $\Phi\lceil\varrho$ is an AND or OR of functions $\Psi_{i}\lceil\varrho$ of decision-tree depth $\leq k$, Lemma 6 implies

$$
\begin{aligned}
\underset{\sigma, \tau}{\mathbb{P}}\left[\mathrm { D } \left(\Phi\left\lceil R_{\alpha q}^{\sigma, \tau}\right) \geq \ell \mid \mathbf{p}^{\sigma, \tau}(\Phi)\right.\right. & \left.=p, R_{p}^{\sigma, \tau}=\varrho \text { and } \mathbf{k}^{\sigma, \tau}(\Phi)=k\right] \\
& =\underset{\theta \sim \mathcal{R}_{\alpha / 14 k}}{\mathbb{P}}\left[\mathrm { D } \left((\Phi\lceil\varrho)\lceil\theta) \geq \ell] \leq\left(5\left(\frac{\alpha}{14 k}\right) k\right)^{\ell} \leq\left(\frac{\alpha}{\mathrm{e}}\right)^{\ell} .\right.\right.
\end{aligned}
$$

\section{Tail bound on $\mathbf{q}(\Phi)$}

Our technical main theorem is a tail bound on the random variable $\mathbf{q}(\Phi)\left(=\mathbf{q}^{\sigma, \tau}(\Phi)\right)$ where the randomness is over independent uniform $\sigma \in\{0,1\}^{n}$ and $\tau \in[0,1]^{n}$. We state the result first with asymptotic notation.

Theorem 10. For every depth $d+1$ formula $\Phi$ and $0<\lambda \leq 1$,

$$
\mathbb{P}[\mathbf{q}(\Phi) \leq \lambda] \leq \frac{|\Phi|}{\exp \left(\Omega\left(d \lambda^{-1 / d}\right)-O(d)\right)}
$$


In order to have a useable induction hypothesis, we restate Theorem 10 with explicit constants:

Theorem 10 (more precisely). For every depth $d+1$ formula $\Phi$ and $\ell>0$,

$$
\mathbb{P}\left[\mathbf{q}(\Phi) \leq \frac{1}{14^{d+1} \ell}\right] \leq|\Phi| \frac{C^{d}}{\exp \left(\mathrm{e}^{-2} d \ell^{1 / d}\right)}
$$

where $C=1+\sum_{i=0}^{\infty}\left(\frac{1}{\exp \left(\mathrm{e}^{i-1}-(i+1) \mathrm{e}^{-2}\right)}+\sum_{j=0}^{\infty} \frac{1}{\exp \left((j+1) \mathrm{e}^{i-1}-(i+j+2) \mathrm{e}^{-2}\right)}\right) \approx 7.83$.

Proof. We first note that the theorem is trivial if $\ell<\mathrm{e}^{d}$ (as the RHS is $>\left(C / \exp \left(\mathrm{e}^{-1}\right)\right)^{d}>1$ since $\left.C>\exp \left(\mathrm{e}^{-1}\right)\right)$. Therefore, we assume that $\ell \geq \mathrm{e}^{d}$. We argue by induction on $d$.

Consider the base case $d=1$ where $\Phi$ is a depth 2 formula. Note that $\mathbf{q}(\Psi)=1 / 14$ for each depth 1 subformula $\Psi$ of $\Phi$; hence $\mathbf{p}(\Phi)=1 / 14$. Also, each $\Psi$ is the AND or OR of decision-trees of depth 1; so by Lemma 6 ,

$$
\underset{\sigma, \tau}{\mathbb{P}}\left[\mathrm{D}\left(\Psi\left\lceil R_{1 / 14}^{\sigma, \tau}\right) \geq \ell\right]=\underset{\varrho \sim \mathcal{R}_{1 / 14}}{\mathbb{P}}\left[\mathrm{D}(\Psi\lceil\varrho) \geq \ell] \leq\left(\frac{1}{\mathrm{e}}\right)^{\ell}\right.\right.
$$

Since $\mathbf{q}(\Phi)=\mathbf{p}(\Phi) / 14 \cdot \mathbf{k}(\Phi)=1 / 14^{2} \cdot \mathbf{k}(\Phi)$, we have

$$
\begin{aligned}
\mathbb{P}\left[\mathbf{q}(\Phi) \leq \frac{1}{14^{2} \ell}\right]=\mathbb{P}[\mathbf{k}(\Phi) \geq \ell] & =\mathbb{P}\left[\bigvee_{\Psi} \mathrm{D}\left(\Psi\left\lceil R_{\mathbf{p}(\Phi)}^{\sigma, \tau}\right) \geq \ell\right]\right. \\
& \leq \sum_{\Psi} \mathbb{P}\left[\mathrm{D}\left(\Psi\left\lceil R_{1 / 14}^{\sigma, \tau}\right) \geq \ell\right]\right. \\
& \leq|\Phi| \frac{1}{\exp (\ell)}<|\Phi| \frac{C^{d}}{\exp \left(\mathrm{e}^{-2} d \ell^{1 / d}\right)}
\end{aligned}
$$

For the induction step, let $d \geq 2$ and assume the theorem holds for $d-1$. Let $\Phi$ be a formula of depth $d+1$. Let $\Psi$ range over depth- $d$ subformulas of $\Phi$. In particular, we have $|\Phi|=1+\sum_{\Psi}|\Psi|$.

We will define a family of events denoted $\mathcal{A}$ and $\mathcal{B}_{i}(i \in \mathbb{N})$ and $\mathcal{C}_{i, j}(i, j \in \mathbb{N})$ and show that the union of these events covers the event $\left\{\mathbf{q}(\Phi) \leq \frac{1}{14^{d+1} \ell}\right\}$. We will then bound the probability of each of these events and show that the (infinite) sum of these probabilities is at most $|\Phi| \frac{C^{d}}{\exp \left(\mathrm{e}^{-2} d \ell^{1 / d}\right)}$.

For all $i \in \mathbb{N}$, define $k_{i}$ and $\alpha_{i}$ by

$$
k_{i}:=\mathrm{e}^{i-1} \ell^{1 / d}, \quad \alpha_{i}:=\frac{k_{i}}{14^{d} \ell}\left(=\frac{1}{14^{d} \mathrm{e}^{1-i} \ell^{(d-1) / d}}\right) .
$$

Events $\mathcal{A}$ and $\mathcal{B}_{i}$ and $\mathcal{C}_{i, j}(i, j \in \mathbb{N})$ are defined as follows:

$$
\begin{aligned}
\mathcal{A} & \stackrel{\text { def }}{\Longleftrightarrow}\left(\mathbf{p}(\Phi) \leq \alpha_{0}\right), \\
\mathcal{B}_{i} & \stackrel{\text { def }}{\Longleftrightarrow} \bigvee_{\Psi}\left(\mathbf{q}(\Psi) \leq \alpha_{i+1}\right) \wedge\left(\mathrm{D}\left(\Psi \uparrow R_{\mathbf{q}(\Psi)}^{\sigma, \tau}\right) \geq k_{i}\right), \\
\mathcal{C}_{i, j} & \stackrel{\text { def }}{\Longleftrightarrow} \bigvee_{\Psi}\left(\alpha_{i+j+1}<\mathbf{q}(\Psi) \leq \alpha_{i+j+2}\right) \wedge\left(\mathrm{D}\left(\Psi \uparrow R_{\alpha_{i+1}}^{\sigma, \tau}\right) \geq k_{i}\right) .
\end{aligned}
$$


$\underline{\text { Claim: }}$ If $\mathbf{q}(\Phi) \leq \frac{1}{14^{d+1} \ell}$, then $\mathcal{A} \vee \bigvee_{i=0}^{\infty}\left(\mathcal{B}_{i} \vee \bigvee_{j=0}^{\infty} \mathcal{C}_{i, j}\right)$

Proof of claim: Assume $\mathbf{q}(\Phi) \leq 1 / 14^{d+1} \ell$ and further assume that $\mathcal{A}$ does not hold. Clearly there exists a unique $i \in \mathbb{N}$ such that $\alpha_{i}<\mathbf{p}(\Phi) \leq \alpha_{i+1}$ (since $\alpha_{i}$ is eventually $>1$ ). Since $\mathbf{q}(\Phi)=\mathbf{p}(\Phi) / 14 \cdot \mathbf{k}(\Phi)$, we have $\mathbf{k}(\Phi)>\alpha_{i} 14^{d} \ell=k_{i}$. Note that $k_{i} \geq k_{0}=\mathrm{e}^{-1} \ell^{1 / d} \geq 1$ (using the assumption that $\left.\ell \geq \mathrm{e}^{d}\right)$. Since $\mathbf{k}(\Phi)=\max \left\{1, \max _{\Psi} \mathrm{D}\left(\Psi\left\lceil R_{\mathbf{p}(\Phi)}^{\sigma, \tau}\right)\right\}\right.$, it follows that there exists a $\Psi$ such that $\mathrm{D}\left(\Psi\left\lceil R_{\mathbf{p}(\Phi)}^{\sigma, \tau}\right) \geq k_{i}\right.$.

Fix an arbitrary choice of $\Psi$ such that $\mathrm{D}\left(\Psi\left\lceil R_{\mathbf{p}(\Phi)}\right) \geq k_{i}\right.$. There are two cases to consider: either $\mathbf{q}(\Psi) \leq \alpha_{i+1}$ or $\alpha_{i+j+1}<\mathbf{q}(\Psi) \leq \alpha_{i+j+2}$ for some $j \in \mathbb{N}$.

- Assume $\mathbf{q}(\Psi) \leq \alpha_{i+1}$. In this case, we have $\mathrm{D}\left(\Psi\left\lceil R_{\mathbf{p}(\Phi)}\right) \leq \mathrm{D}\left(\Psi\left\lceil R_{\mathbf{q}(\Psi)}\right)\right.\right.$ since $\mathbf{p}(\Phi) \leq \mathbf{q}(\Psi)$. Therefore, $\mathrm{D}\left(\Psi\left\lceil R_{\mathbf{q}(\Psi)}^{\sigma, \tau}\right) \geq k_{i}\right.$. We conclude that $\mathcal{B}_{i}$ holds.

- Assume $\alpha_{i+j+1}<\mathbf{q}(\Psi) \leq \alpha_{i+j+2}$ for some $j \in \mathbb{N}$. We have $\mathrm{D}\left(\Psi\left\lceil R_{\mathbf{p}(\Phi)}\right) \leq \mathrm{D}\left(\Psi\left\lceil R_{\alpha_{i+1}}\right)\right.\right.$ since $\mathbf{p}(\Phi) \leq \alpha_{i+1}$. Therefore, $\mathrm{D}\left(\Psi\left\lceil R_{\alpha_{i+1}}^{\sigma, \tau}\right) \geq k_{i}\right.$. We conclude that $\mathcal{C}_{i, j}$ holds.

This concludes the proof of the claim.

To complete the proof of the theorem, we will bound the probabilities of events $\mathcal{A}, \mathcal{B}_{i}$ and $\mathcal{C}_{i, j}$ and take a union bound. We ignore the fact that all but finitely many of these events have zero probability, since $\mathbb{P}\left[\mathcal{B}_{i}\right]=0$ (resp. $\mathbb{P}\left[\mathcal{C}_{i, j}\right]=0$ ) for all $\alpha_{i}>1\left(\right.$ resp. $\left.\alpha_{i+j+1}>1\right)$. Instead, we show that $\mathbb{P}\left[\mathcal{B}_{i}\right]$ is exponentially decreasing in $i$, while $\operatorname{Pr}\left[\mathcal{C}_{i, j}\right]$ is exponentially decreasing in $j$ and doubly exponentially decreasing in $i$.

We first bound the probability of $\mathcal{A}$ :

$$
\begin{aligned}
\mathbb{P}[\mathcal{A}]=\mathbb{P}\left[\bigvee_{\Psi} \mathbf{q}(\Psi) \leq \frac{1}{14^{d} \mathrm{e} \ell^{(d-1) / d}}\right] & \leq \sum_{\Psi} \mathbb{P}\left[\mathbf{q}(\Psi) \leq \frac{1}{14^{d} \mathrm{e}^{(d-1) / d}}\right] \\
& \leq|\Phi| \frac{C^{d-1}}{\exp \left(\mathrm{e}^{-2}(d-1) \mathrm{e}^{1 /(d-1)} \ell^{1 / d}\right)} \quad \text { (induction hypothesis) } \\
& \leq|\Phi| \frac{C^{d-1}}{\exp \left(\mathrm{e}^{-2} d \ell^{1 / d}\right)} \quad\left(\text { using } \mathrm{e}^{1 /(d-1)} \geq \frac{d}{d-1}\right) .
\end{aligned}
$$


We next bound the probability of $\mathcal{B}_{i}$ :

$$
\begin{aligned}
\mathbb{P}\left[\mathcal{B}_{i}\right] & =\mathbb{P}\left[\bigvee_{\Psi}\left(\mathbf{q}(\Psi) \leq \alpha_{i+1}\right) \wedge\left(\mathrm{D}\left(\Psi \uparrow R_{\mathbf{q}(\Psi)}^{\sigma, \tau}\right) \geq k_{i}\right)\right] \\
& \leq \sum_{\Psi} \mathbb{P}\left[\mathbf{q}(\Psi) \leq \alpha_{i+1}\right] \mathbb{P}\left[\mathrm{D}\left(\Psi \uparrow R_{\mathbf{q}(\Psi)}^{\sigma, \tau}\right) \geq k_{i} \mid \mathbf{q}(\Psi) \leq \alpha_{i+1}\right] \\
& \leq\left(\frac{1}{\mathrm{e}}\right)^{k_{i}} \sum_{\Psi} \mathbb{P}\left[\mathbf{q}(\Psi) \leq \alpha_{i+1}\right] \quad \text { (Lemma 9) } \\
& =\frac{1}{\exp \left(\mathrm{e}^{i-1} \ell^{1 / d}\right)} \sum_{\Psi} \mathbb{P}\left[\mathbf{q}(\Psi) \leq \frac{1}{14^{d} \mathrm{e}^{-i} \ell^{(d-1) / d}}\right] \\
& \leq \frac{1}{\exp \left(\mathrm{e}^{i-1} \ell^{1 / d}\right)}|\Phi| \frac{C^{d-1}}{\exp \left(\mathrm{e}^{-2}(d-1) \mathrm{e}^{-i /(d-1)} \ell^{1 / d}\right)} \\
& \leq \frac{1}{\exp \left(\mathrm{e}^{i-1} \ell^{1 / d}\right)}|\Phi| \frac{C^{d-1}}{\exp \left(\mathrm{e}^{-2}(d-1) \ell^{1 / d}-i \mathrm{e}^{-2} \ell^{1 / d}\right)} \\
& =\frac{1}{\exp \left(\left(\mathrm{e}^{i-1}-(i+1) \mathrm{e}^{-2}\right) \ell^{1 / d}\right)}|\Phi| \frac{C^{d-1}}{\exp \left(\mathrm{e}^{-2} d \ell^{1 / d}\right)} \\
& \leq \frac{\left.\mathrm{e}^{-i /(d-1)} \geq 1-\frac{i}{d-1}\right)}{\exp \left(\mathrm{e}^{i-1}-(i+1) \mathrm{e}^{-2}\right)}|\Phi| \frac{C^{d-1}}{\exp \left(\mathrm{e}^{-2} d \ell^{1 / d}\right)}
\end{aligned}
$$

The last inequality uses the assumption $\ell^{1 / d} \geq 1$ as well as the nonnegativity of $\mathrm{e}^{i-1}-(i+1) \mathrm{e}^{-2}$ for all $i \in \mathbb{N}$.

Finally, we bound the probability of $\mathcal{C}_{i, j}$ :

$$
\begin{aligned}
& \mathbb{P}\left[\mathcal{C}_{i, j}\right]=\mathbb{P}\left[\bigvee_{\Psi}\left(\alpha_{i+j+1}<\mathbf{q}(\Psi) \leq \alpha_{i+j+2}\right) \wedge\left(\mathrm{D}\left(\Psi \mid R_{\alpha_{i+1}}^{\sigma, \tau}\right) \geq k_{i}\right)\right] \\
& \leq \sum_{\Psi} \mathbb{P}\left[\mathbf{q}(\Psi) \leq \alpha_{i+j+2}\right] \mathbb{P}\left[\mathrm{D}\left(\Psi \mid R_{\alpha_{i+1}}^{\sigma, \tau}\right) \geq k_{i} \mid \alpha_{i+j+1}<\mathbf{q}(\Psi) \leq \alpha_{i+j+2}\right] \\
& \leq\left(\frac{\alpha_{i+1} / \alpha_{i+j+1}}{\mathrm{e}}\right)^{k_{i}} \sum_{\Psi} \mathbb{P}\left[\mathbf{q}(\Psi) \leq \alpha_{i+j+2}\right] \\
& =\frac{1}{\exp \left((j+1) \mathrm{e}^{i-1} \ell^{1 / d}\right)} \sum_{\Psi} \mathbb{P}\left[\mathbf{q}(\Psi) \leq \frac{1}{14^{d} \mathrm{e}^{-(i+j+1)} \ell^{(d-1) / d}}\right] \\
& \left.\leq \frac{1}{\exp \left((j+1) \mathrm{e}^{i-1} \ell^{1 / d}\right)}|\Phi| \frac{C^{d-1}}{\exp \left(\mathrm{e}^{-2}(d-1) \mathrm{e}^{-(i+j+1) /(d-1)} \ell^{1 / d}\right)} \quad \text { (ind. hyp. }\right) \\
& \leq \frac{1}{\exp \left((j+1) \mathrm{e}^{i-1} \ell^{1 / d}\right)}|\Phi| \frac{C^{d-1}}{\exp \left(\mathrm{e}^{-2}(d-1) \ell^{1 / d}-(i+j+1) \mathrm{e}^{-2} \ell^{1 / d}\right)} \\
& =\frac{1}{\exp \left(\left((j+1) \mathrm{e}^{i-1}-(i+j+2) \mathrm{e}^{-2}\right) \ell^{1 / d}\right)}|\Phi| \frac{C^{d-1}}{\exp \left(\mathrm{e}^{-2} d \ell^{1 / d}\right)} \\
& \leq \frac{1}{\exp \left((j+1) \mathrm{e}^{i-1}-(i+j+2) \mathrm{e}^{-2}\right)}|\Phi| \frac{C^{d-1}}{\exp \left(\mathrm{e}^{-2} d \ell^{1 / d}\right)} \text {. }
\end{aligned}
$$

The last inequality uses the assumption $\ell^{1 / d} \geq 1$ and the nonnegativity of $(j+1) \mathrm{e}^{i-1}-(i+j+2) \mathrm{e}^{-2}$ for all $i, j \in \mathbb{N}$. 
We finish the proof by taking a union bound:

$$
\mathbb{P}\left[\mathbf{q}(\Phi) \leq \frac{1}{14^{d+1} \ell}\right] \leq \mathbb{P}[\mathcal{A}]+\sum_{i=0}^{\infty}\left(\mathbb{P}\left[\mathcal{B}_{i}\right]+\sum_{j=0}^{\infty} \mathbb{P}\left[\mathcal{C}_{i, j}\right]\right) \leq|\Phi| \frac{C^{d}}{\exp \left(\mathrm{e}^{-2} d \ell^{1 / d}\right)}
$$

\section{PARITY}

We use the results of the last section to prove our lower bound for the PARITY function.

Theorem 3 (restated). Depth $d+1$ formulas computing PARITY require size $\exp \left(\Omega\left(d\left(n^{1 / d}-1\right)\right)\right)$.

Proof. Suppose $\Phi$ is a depth $d+1$ formula computing PARITY. Then

$$
\underset{\varrho \sim \mathcal{R}_{1 / n}}{\mathbb{P}}\left[\Phi\lceil\varrho \text { is non-constant }]=1-\left(1-\frac{1}{n}\right)^{n}>1-\frac{1}{\mathrm{e}} .\right.
$$

On the other hand, by Theorem 10 and Lemma 9 ,

$$
\begin{aligned}
\underset{\varrho \sim \mathcal{R}_{1 / n}}{\mathbb{P}}[\Phi\lceil\varrho \text { is non-constant }] & =\underset{\sigma, \tau}{\mathbb{P}}\left[\mathrm{D}\left(\Phi\left\lceil R_{1 / n}^{\sigma, \tau}\right) \geq 1\right]\right. \\
& \leq \mathbb{P}\left[\mathrm { D } \left(\Phi\left\lceil R_{\max \{1 / n, \mathbf{q}(\Phi)\}}^{\sigma, \tau} \geq 1\right]\right.\right. \\
& \leq \mathbb{P}[\mathbf{q}(\Phi) \leq 1 / n]+\mathbb{P}\left[\mathrm{D}\left(\Phi\left\lceil R_{\mathbf{q}(\Phi)}^{\sigma, \tau}\right) \geq 1\right]\right. \\
& \leq \frac{|\Phi|}{\exp \left(\Omega\left(d n^{1 / d}\right)-O(d)\right)}+\frac{1}{\mathrm{e}}
\end{aligned}
$$

Therefore,

$$
|\Phi| \geq\left(1-\frac{2}{\mathrm{e}}\right) \exp \left(\Omega\left(d n^{1 / d}\right)-O(d)\right)
$$

It follows that there exist universal constants $c_{0}, c_{1}>0$ (determined by the constants in the $\Omega(\cdot)$ and $O(\cdot))$ such that $|\Phi| \geq \exp \left(c_{0} d\left(n^{1 / d}-1\right)\right)$ in the regime $d \leq c_{1} \ln n$.

In the regime $d>c_{1} \ln n$, we have $d\left(n^{1 / d}-1\right)=\Theta(\ln n)$, more precisely,

$$
\ln n<d\left(n^{1 / d}-1\right)<c_{1}\left(e^{c_{1}}-1\right) \ln n .
$$

Note that $d\left(n^{1 / d}-1\right)$ is decreasing in $d$ and $\lim _{d \rightarrow \infty} d\left(n^{1 / d}-1\right)=\ln n$. Invoking Khrapchenko's $n^{2}$ leafsize lower bound [5] (which implies a (gate)size lower bound of $n$ ), we get a tight lower bound of $\exp \left(\Omega\left(d\left(n^{1 / d}-1\right)\right)\right)$ which is valid for all $d$ and $n$.

\section{Average Sensitivity}

Theorem 4 (restated). Depth $d+1$ formulas of size s have average sensitivity $O\left(\frac{1}{d} \ln s\right)^{d}$.

Proof. Let $\Phi$ be a formula of depth $d+1$ and size $s$ (recall that size is the number of gates). Assume $\operatorname{as}(\Phi) \geq 1$, since otherwise the theorem is trivial. We further assume that $\Phi$ has bottom fan-in $\leq s$; otherwise it is easily shown that as $(\Phi)=O\left(\operatorname{as}\left(\Phi^{\prime}\right)\right)$ where $\Phi^{\prime}$ is obtained from $\Phi$ by replacing every bottom AND (resp. OR) gate with fan-in $>s$ with 0 (resp. 1). In particular, $\Phi$ has leafsize $\leq s^{2}$, so it depends on $\leq s^{2}$ distinct variables. 
Letting $p=1 / \operatorname{as}(\Phi)$ and using facts (11) and (2), we have

$$
1=p \cdot \operatorname{as}(\Phi)=\underset{\varrho \sim \mathcal{R}_{p}}{\mathbb{E}}\left[\operatorname{as}(\Phi\lceil\varrho)] \leq \underset{\sigma, \tau}{\mathbb{E}}\left[\mathrm{D}\left(\Phi\left\lceil R_{p}^{\sigma, \tau}\right)\right]=\sum_{k=1}^{s^{2}} \underset{\sigma, \tau}{\mathbb{P}}\left[\mathrm{D}\left(\Phi\left\lceil R_{p}^{\sigma, \tau}\right) \geq k\right] .\right.\right.\right.
$$

For all $k \in \mathbb{N}$, by Theorem 10 and Lemma 9 ,

$$
\begin{aligned}
\underset{\sigma, \tau}{\mathbb{P}}\left[\mathrm{D}\left(\Phi\left\lceil R_{p}^{\sigma, \tau}\right) \geq k\right]\right. & \leq \underset{\sigma, \tau}{\mathbb{P}}\left[\mathrm{D}\left(\Phi\left\lceil R_{\max \{p, \mathbf{q}(\Phi)\}}^{\sigma, \tau}\right) \geq k\right]\right. \\
& \leq \mathbb{P}[\mathbf{q}(\Phi) \leq p]+\mathbb{P}\left[\mathrm{D}\left(\Phi\left\lceil R_{\mathbf{q}(\Phi)}^{\sigma, \tau}\right) \geq k\right]\right. \\
& \leq \frac{s}{\exp \left(\Omega\left(d \cdot \operatorname{as}(\Phi)^{1 / d}\right)-O(d)\right)}+\frac{1}{\mathrm{e}^{k}} .
\end{aligned}
$$

Combining these inequalities, we have

$$
\exp \left(\Omega\left(d \cdot \operatorname{as}(\Phi)^{1 / d}\right)-O(d)\right) \leq \frac{s^{3}}{1-\sum_{k=1}^{\infty} \mathrm{e}^{-k}}=\frac{1-\mathrm{e}^{-1}}{1-2 \mathrm{e}^{-1}} s^{3}=O\left(s^{3}\right)
$$

It follows that $\Omega\left(d \cdot \operatorname{as}(\Phi)^{1 / d}\right) \leq 3 \ln s+O(d)$ and therefore as $(\Phi)=O\left(\frac{1}{d} \ln s\right)^{d}$.

\section{Formulas vs. Circuits}

Our lower bound for PARITY (Theorem [3) implies a separation between the power of depth $d+1$ formulas vs. circuits. We write \{poly-size depth $d+1$ circuits/formulas for the non-uniform complexity class of languages computable by $n^{O(1)}$-size depth $d+1$ circuits/formulas where $d(n)$ is an arbitrary function of $n$.

Corollary 11. For all $d(n)=o(\log n)$ with $\lim _{n \rightarrow \infty} d(n)=\infty$,

$$
\{\text { poly-size depth } d+1 \text { formulas }\} \neq\{\text { poly-size depth } d+1 \text { circuits }\} \text {. }
$$

Moreover, for all $d \leq C \frac{\log n}{\log \log n}$ (for some universal constant $C>0$ ),

$$
\{\text { poly-size depth } d+1 \text { circuits }\} \nsubseteq\left\{n^{o(d)} \text {-size depth } d+1 \text { formulas }\right\} \text {. }
$$

Separation (3) may be regarded as the depth $d+1$ analogue of the conjectured separation $\{$ poly-size formulas $\} \neq\{$ poly-size circuits $\}$, also known as $\mathrm{NC}^{1} \neq \mathrm{P} /$ poly. By Spira's theorem [7, every poly-size formula is equivalent to a poly-size formula of depth $O(\log n)$; thus, extending (3) from depth $o(\log n)$ to depth $O(\log n)$ would imply $N C^{1} \neq \mathrm{P} /$ poly (in fact $\left.N C^{1} \neq \mathrm{AC}^{1}\right)$.

For the smaller range of $d \leq c \frac{\log n}{\log \log n}$, we get the stronger separation (44). In light of Fact 5 , this is the strongest possible separation between formulas and circuits of the same depth.

We remark that until recently not even the weak separation (3) was known to hold for any super-constant $d \not \leq O(1)$. The first progress on this question was made in [6], where (4) was shown to hold for all $d \leq \log \log \log n$ via a lower bound for DISTANCE- $\log \log n$ ST-CONNECTIVITY. In fact, the lower bound of [6] implies a much stronger result: for all $d \leq \log \log \log n$,

$$
\text { \{poly-size depth } d+1 \text { circuits }\} \nsubseteq\left\{n^{o(d)} \text {-size depth } \frac{\log n}{(\log \log n)^{3}} \text { formulas }\right\} .
$$

It remains an open problem to push separation (5) to greater depths. 


\section{Acknowledgements}

My thanks to Rahul Santhanam, Rocco Servedio and Li-Yang Tan for valuable discussions and to the anonymous referees of FOCS'15 for their helpful feedback. This work was carried out while the author was a research fellow at the Simons Institute.

\section{References}

[1] Miklós Ajtai. $\Sigma_{1}^{1}$ formulae on finite structures. Annals of Pure and Applied Logic, 24:1-48, 1983.

[2] Ravi B. Boppana. The average sensitivity of bounded-depth circuits. Information Processing Letters, 63(5):257-261, 1997.

[3] Merrick L. Furst, James B. Saxe, and Michael Sipser. Parity, circuits, and the polynomial-time hierarchy. Mathematical Systems Theory, 17:13-27, 1984.

[4] Johan Håstad. Almost optimal lower bounds for small depth circuits. In 18th Annual ACM Symposium on Theory of Computing, pages 6-20, 1986.

[5] V.M. Khrapchenko. Complexity of the realization of a linear function in the case of П-circuits. Math. Notes Acad. Sciences, 9:21-23, 1971.

[6] Benjamin Rossman. Formulas vs. circuits for small distance connectivity. In 46th Annual ACM Symposium on Theory of Computing, pages 203-212, 2014.

[7] P.M. Spira. On time-hardware complexity tradeoffs for Boolean functions. In 4th Hawaii Symposium on System Sciences, pages 525-527, 1971.

[8] Andrew C.C. Yao. Separating the polynomial-time hierarchy by oracles. In 26th Annual IEEE Symposium on Foundations of Computer Science, pages 1-10, 1985. 